\title{
Enhancing Acceleration Radiation from Ground- State Atoms via Cavity Quantum Electrodynamics
}

\section{Citation}

Scully, Marlan 0., Vitaly V. Kocharovsky, Alexey Belyanin, Edward Fry, and Federico Capasso. 2003. "Enhancing Acceleration Radiation from Ground-State Atoms via Cavity Quantum Electrodynamics." Physical Review Letters 91 (24). https://doi.org/10.1103/ physrevlett.91.243004.

\section{Permanent link}

http://nrs.harvard.edu/urn-3:HUL.InstRepos:41372670

\section{Terms of Use}

This article was downloaded from Harvard University's DASH repository, and is made available under the terms and conditions applicable to Other Posted Material, as set forth at http:// nrs.harvard.edu/urn-3:HUL.InstRepos:dash.current.terms-of-use\#LAA

\section{Share Your Story}

The Harvard community has made this article openly available. Please share how this access benefits you. Submit a story.

Accessibility 


\title{
Enhancing Acceleration Radiation from Ground-State Atoms via Cavity Quantum Electrodynamics
}

\author{
Marlan O. Scully, ${ }^{1,2}$ Vitaly V. Kocharovsky, ${ }^{1,3}$ Alexey Belyanin,,${ }^{1,3}$ Edward Fry, ${ }^{1}$ and Federico Capasso ${ }^{4}$ \\ ${ }^{1}$ Institute for Quantum Studies and Department of Physics, Texas A $8 M$ Univ., TX 77843, USA \\ ${ }^{2}$ Max-Planck-Institut für Quantenoptik, D-85748 Garching, Germany \\ ${ }^{3}$ Institute of Applied Physics RAS, 603950 Nizhny Novgorod, Russia \\ ${ }^{4}$ Division of Engineering and Applied Sciences, Harvard University, Cambridge, Massachusetts, USA
}

\begin{abstract}
When ground state atoms are accelerated through a high Q microwave cavity, radiation is produced with an intensity which can exceed the intensity of Unruh acceleration radiation in free space by many orders of magnitude. The cavity field at steady state is described by a thermal density matrix under most conditions. However, under some conditions gain is possible, and when the atoms are injected in a regular fashion, the radiation can be produced in a squeezed state.
\end{abstract}

One of the most intriguing results of modern quantum field theory is the proof by Davies, Fulling, Unruh and DeWitt [1], and others [2] that ground state atoms, accelerated through vacuum, are promoted to an excited state just as if they were in contact with a blackbody thermal field. These studies $[1,2]$ predict that a (twolevel) ground state atom, having transition frequency $\omega$, and experiencing a constant acceleration $a$, will be excited to its upper level with a probability governed by the Boltzmann factor $\exp (-2 \pi \omega / \alpha)$, where $\alpha=a / c, c$ is the speed of light in vacuum. Unfortunately, even for very large acceleration "frequency" $\alpha \approx 10^{8} \mathrm{~Hz}[3]$, and microwave frequency $\omega \approx 10^{10} \mathrm{~Hz}[4]$, this factor is exponentially small, $\sim 10^{-200}$; and is not of experimental interest.

Thus we were motivated to study a simple gedanken experiment based on a model consisting of a high Q "single mode" cavity through which we pass accelerated twolevel atoms as in Fig. [1 We find that the radiation is thermal (in the typical case) and the effective "Boltzmann factor" is now given by $\alpha / 2 \pi \omega$. For the above example, $\alpha / 2 \pi \omega \sim 10^{-3}$, hence, it is many orders of magnitude larger than that for the usual Unruh effect and is potentially observable.

The envisioned experiment can be described as a kind of "acceleration radiation" mazer [5, 6]. In the ordinary maser, stimulated emission is the mechanism for the production of radiation. In the present case, the physics of the emission process is intimately association with the center-of-mass motion (taken in the $z$ direction).

One scheme for accelerating [7] the atoms uses a particle accelerator with, e.g., hydrogen like ions. In such a case, ordinary (i.e. not Unruh) radiation emitted by accelerated charged particles must be taken into account. Alternatively, we could envision atoms accelerated in a strong gravitational field through a cavity. Other means of operation via periodically driven atoms are also possible as in Figs. 1(b,c) and are discussed later. For the moment, we simply assume the trajectories given by, e.g., Eq. (2) and neglect the quantization of translational motion and recoil effect.

Our main results are contained in Eqs. (4)-(9). We find that the acceleration radiation is generated by a kind of

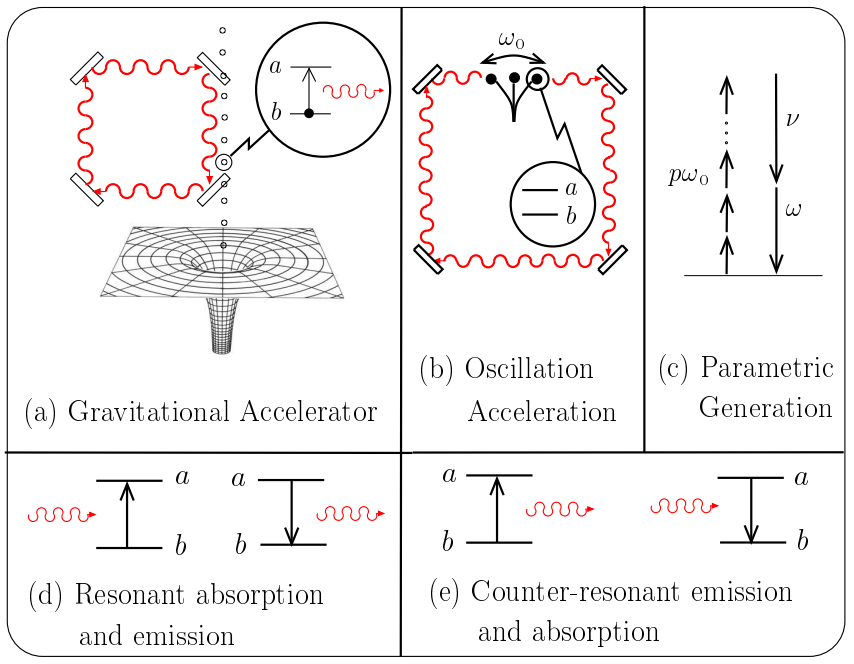

FIG. 1: (a) Atoms in the ground state $|b\rangle$ are accelerated through small holes in the corner reflectors of a microwave (or optical) cavity by, e.g., a strong gravitational field. This is depicted as a unidirectional, single mode, ring cavity to convey the idea. (b) "Vibrating reed" piezoelectrically driven oscillator containing a two-level atom is placed in the cavity yielding strong mazer action . (c) Parametric conversion of vibronic energy $p \hbar \omega_{\text {o }}$ into photon and atom energies $\hbar \nu$ and $\hbar \omega$ respectively. (d) An atom is excited (de-excited) as it simultaneously absorbs (emits) a photon in a resonant process. (e) The counter-resonant processes that are usually neglected as compared to the resonant processes in the "rotating wave" approximation; i.e. an atom is excited (de-excited) as it simultaneously emits (absorbs) a photon.

parametric process [8] in which both the atomic polarization (the idler) and the radiation (the signal) are excited by extracting energy from the atomic center-of-mass motion (the pump). Such processes are intimately related to the so-called counter-rotating terms in the atom-field interaction Hamiltonian and are discarded in the rotating wave approximation (RWA).

This provides a simple picture for the generation of acceleration radiation. The photons emitted are real. The generation of radiation by the counter-rotating terms is interesting; but, perhaps, no more bizarre than the ear- 
lier demonstration of mazer emission 5 due to scattering of atoms off the cavity interface 9]. Furthermore, we find that the radiation may even be squeezed when $S_{1,2}$ in Eq. (8) are nonvanishing. Calculation details will be given elsewhere [10] as will experimental implications 11.

As in the quantum theory of the laser 12, 13], the (microscopic) change in the density matrix of a cavity mode due to any one atom, $\delta \rho^{i}$, is small. The (macroscopic) change due to $\Delta N$ atoms is then $\Delta \rho=\sum_{i} \delta \rho^{i}=\Delta N \delta \rho$. Writing $\Delta N=r \Delta t$, where $r$ is the atomic injection rate, we have a coarse grained equation of motion: $\Delta \rho / \Delta t=$ $r \delta \rho$. The change $\delta \rho^{i}$ due to an atom injected at time $\tau_{i}$ in the atomic rest frame is

$$
\begin{array}{r}
\delta \rho^{i}=-\frac{1}{\hbar^{2}} \int_{\tau_{i}}^{\tau_{i}+T} \int_{\tau_{i}}^{\tau_{i}+\tau^{\prime}} \operatorname{tr}_{\text {atom }} \times \\
\times\left[\hat{V}\left(\tau^{\prime}\right),\left[\hat{V}\left(\tau^{\prime \prime}\right), \rho^{\text {atom }}\left(\tau_{i}\right) \otimes \rho\left(t\left(\tau_{i}\right)\right)\right]\right] d \tau^{\prime} d \tau^{\prime \prime},
\end{array}
$$

where $T$ is the proper time of flight through the cavity and $\operatorname{tr}_{\text {atom }}$ denotes the trace over atom states. The time $\tau$ is the atomic proper time, i.e., the time measured by an observer riding along with the atom. The cavity proper time $t(\tau)$ and the atomic trajectory of the atom as it passes through the cavity, $z(\tau)$, are given by 14 ]

$$
t(\tau)=t_{0}+\frac{1}{\alpha} \sinh (\alpha \tau), \quad \mathrm{z}(\tau)=\frac{\mathrm{c}}{\alpha}[\cosh (\alpha \tau)-1],
$$

where $t_{0}=t(\tau=0)$ is the moment of time in the laboratory (cavity) frame when the atom starts its acceleration. The distinction between atomic and cavity field proper times is important. It is most convenient to calculate $\delta \rho^{i}$ in the atomic frame. In the case of a running wave with a wave vector $\mathbf{k}, k_{z}=\mathbf{k} \cdot \mathbf{v} / v$, the atom-field interaction Hamiltonian in the atomic frame is given by

$$
\hat{V}(\tau)=\hbar g(\tau)\left[\hat{a}_{k} e^{-i \nu t(\tau)+i k_{z} z(\tau)}+\text { h.c. }\right]\left[\hat{\sigma} e^{-i \omega \tau}+\text { h.c. }\right]
$$

Here $g(\tau)=\mu E^{\prime} / \hbar$ is the atom-field coupling frequency which depends on the atomic dipole moment $\mu$ and the electrical field $E^{\prime}$ in the frame of the atom. For simplicity, consider the case of the co-propagating atom and field, $k_{z}=|\mathbf{k}|=\nu / c$, so that $E^{\prime}=\sqrt{(c-v) /(c+v)} E$. Since $v=c \tanh (\alpha \tau)$ for a uniformly accelerated particle, we have $E^{\prime}=\mathrm{e}^{-\alpha \tau} E$ and $g(\tau)=g \mathrm{e}^{-\alpha \tau}$. The operator $\hat{a}_{k}$ is the annihilation operator for the running wave, while $\hat{\sigma}$ is the atomic lowering operator. Inserting Eq. (3) into Eq. (1) and using Eq. (2), we obtain 10] the results given in Eqs. (4)-(8) below.

In the case of random injection times, the equation of motion for the density matrix of the field is

$$
\begin{aligned}
d \rho_{n, n} / d t= & -R_{2}\left[(n+1) \rho_{n, n}-n \rho_{n-1, n-1}\right] \\
& -R_{1}\left[n \rho_{n, n}-(n+1) \rho_{n+1, n+1}\right],
\end{aligned}
$$

where $R_{1,2}$ are defined in the following. If $R_{1}>R_{2}$, there is a steady state solution which is thermal [12]

$$
\rho_{n, n}=e^{-\hbar \nu n / k_{B} \mathcal{T}_{c}}\left(1-\mathrm{e}^{-\hbar \nu / k_{B} \mathcal{T}_{c}}\right)
$$

$$
\bar{n}=\sum_{n} n \rho_{n n}=\frac{1}{\mathrm{e}^{\hbar \nu / k_{B} \mathcal{T}_{c}}-1}, \mathrm{e}^{-\hbar \nu / k_{B} \mathcal{T}_{c}}=\frac{R_{2}}{R_{1}},
$$

where an effective temperature of the field in the cavity is $\mathcal{T}_{c}=\hbar \nu / k_{B} \ln \left[R_{1} / R_{2}\right]$. Thus, spontaneous emission of randomly injected ground state atoms in the cavity results in thermal statistics of the mode excitation. Note, that the thermal statistics of the atomic excitation in the standard Unruh effect in free space is due to spontaneous emission into a vacuum field reservoir with a continuous spectrum of modes.

Absorption and emission coefficients $R_{1,2}=r\left|g I_{1,2}\right|^{2}$ are determined by the amplitude $g \mathrm{e}^{-i \nu / \alpha} I_{1,2}=$ $-\frac{i}{\hbar} \int_{\tau_{i}}^{\tau_{i}+T} V_{1,2} d \tau$ of the matrix elements $V_{1}=\langle a, 0|\hat{V}| b, 1\rangle$ and $V_{2}=\langle a, 1|\hat{V}| b, 0\rangle$ of the interaction Hamiltonian (3), respectively. In particular case $\tau_{i}=0$ we find

$$
I_{1}(\omega)=\int_{0}^{T} \exp \left[i \frac{\nu}{\alpha} \mathrm{e}^{-\alpha \tau}+i \omega \tau-\alpha \tau\right] d \tau
$$

It is convenient to write this as

$$
I_{1}(\omega)=\left[\int_{\tau^{*}}^{T} d \tau-\int_{\tau^{*}}^{0} d \tau\right] \exp \left[i \frac{\nu}{\alpha} \mathrm{e}^{-\alpha \tau}+i \omega \tau-\alpha \tau\right],
$$

where $\tau^{*}=-\infty-i \pi / 2 \alpha$. We carry out the first integral by changing the variable of integration to $x=$ $-i(\nu / \alpha) \mathrm{e}^{-\alpha \tau}$ and assume that $\mathrm{e}^{-\alpha T} \approx 0$. In such a case the first integral is proportional to the ordinary gamma function defined as $\Gamma(z)=\int_{0}^{\infty} \mathrm{e}^{-x} x^{z-1} d x$. The second integral may be adequately approximated by integration by parts in the limit that $\frac{\alpha}{\nu} \ll 1$ and $\frac{\alpha}{\omega} \ll 1$. We find

$$
\begin{array}{r}
I_{1}(\omega)=\frac{i}{\nu}\left(\frac{\alpha}{\nu}\right)^{-i \frac{\omega}{\alpha}} \mathrm{e}^{\frac{\pi \omega}{2 \alpha}} \Gamma\left(1-\frac{i \omega}{\alpha}\right) \\
-\frac{i \mathrm{e}^{i \frac{\nu}{\alpha}}}{\nu-\omega}\left[1+\mathrm{O}\left(\frac{\alpha \omega}{(\nu-\omega)^{2}}\right)\right] .
\end{array}
$$

The corresponding integral for the emission of radiation $I_{2}(\omega)$ is equal to $I_{1}(-\omega)$. We proceed to calculate $R_{1} \propto\left|I_{1}(\omega)\right|^{2}$ and $R_{2} \propto\left|I_{2}(\omega)\right|^{2}$ by noting that $\Gamma(1+i \omega / \alpha) \Gamma(1-i \omega / \alpha)=(\pi \omega / \alpha) / \sinh (\pi \omega / \alpha)$.

We find that in the limit $\nu \gg \omega \gg \alpha$ the emission/absorption ratio is $R_{2} / R_{1} \simeq \alpha /(2 \pi \omega)$, which is an enhancement by many orders of magnitude as compared to the exponentially small value $R_{2} / R_{1}=\exp (-2 \pi \omega / \alpha)$.

For arbitrary values of parameters, the absorption and emission amplitudes can be calculated as

$$
I_{1,2}(\omega)=\frac{i}{\nu}\left(\frac{\alpha}{\nu}\right)^{\mp i \frac{\omega}{\alpha}} \mathrm{e}^{ \pm \frac{\pi \omega}{2 \alpha}}\left[\Gamma\left(z, u \mathrm{e}^{-\alpha T}\right)-\Gamma(z, u)\right]
$$

where $z=1 \mp i \frac{\omega}{\alpha}, u=-i \frac{\nu}{\alpha} \mathrm{e}^{-\alpha \tau_{i}}$, and $\Gamma(z, u)=$ $\int_{u}^{\infty} \mathrm{e}^{-x} x^{z-1} d x$ is the incomplete gamma function. 
The above analysis clearly shows that the mechanism of the field and atom excitation in cavity quantum electrodynamics is the same as for the Unruh effect in free space and is nothing but a nonadiabatic transition due to the counter-rotating term $\hat{a}_{k}^{+} \hat{\sigma}^{+}$in the interaction Hamiltonian (3), i.e. $V_{2}$. The reason for an enhanced excitation in the cavity is the relatively large amplitude for a quantum transition $|b, 0\rangle \rightarrow|a, 1\rangle$ due to the sudden nonadiabatic switching on of the interaction. As a result of this rapid turn on, the initial state $|b, 0\rangle$ is no longer an eigenstate of the Hamiltonian. Now, a linear superposition of the excited states of atom and field makes up the dressed [14] ground state of the interacting system $\psi_{0}=|b, 0\rangle-\frac{g(\tau)}{\nu^{\prime}+\omega}|a, 1\rangle$ as well as the dressed excited state $\psi_{1}=|a, 1\rangle+\frac{g(\tau)}{\nu^{\prime}+\omega}|b, 0\rangle$.

In particular, the amplitude of the bare excited state $|a, 1\rangle$ in $\psi_{0}$ is of the order of $C \sim \mu E^{\prime} / \hbar\left(\omega+\nu^{\prime}\right)$. The latter corresponds to the atomic excitation probability $\rho_{\text {aa }}^{\text {atom }}=|C|^{2} \sim\left|\mu E^{\prime} / \hbar(\omega+\nu)\right|^{2} \sim\left|g I_{2}\right|^{2}$. This can be also found directly from the density matrix equation for the atom, via the atomic counterpart to Eq. (1) with a trace over the photon states instead of the $\operatorname{tr}_{\text {atom. This }}$ probability has the same origin and value as the wellknown Bloch-Siegert shift of a two-level atomic transition [14], $\Delta \omega / \omega=\left(\mu E^{\prime} / \hbar(\omega+\nu)\right)^{2}$, due to counter-rotating terms in the interaction Hamiltonian.

Clearly, the second term in Eqs. (6b) and (7a) represents the contributions from boundaries to the nonadiabatic transition amplitudes. In the absence of the boundary contributions, the emission integral $I_{2}(\omega)=$ $I_{1}(-\omega)$ in Eqs. (6) and (7) becomes exponentially small $\sim \exp (-\pi \omega / \alpha)$ for the small parameter $\alpha / 2 \pi \omega \ll 1$ since there are no stationary phase points in the integration interval. The absorption integral $I_{1}$ does have a point of stationary phase when the atomic frequency $\omega$ is brought into resonance with the field due to the time-dependent Doppler shift of the mode frequency 16 $\nu^{\prime}(\tau)=\nu \exp (-\alpha \tau)$. This fact explains why the related exponential factor effectively disappears from the absorption integral $(7 \mathrm{a}),\left|\mathrm{e}^{\frac{\pi \omega}{2 \alpha}} \Gamma(1-i \omega / \alpha)\right| \simeq(2 \pi \omega / \alpha)^{1 / 2}$, when $\alpha \ll 2 \pi \omega$. As a result, if there are no edge effects, we obtain the same excitation factor $R_{2} / R_{1}=\exp (-2 \pi \omega / \alpha)$ as in the Unruh effect (in free space). This means that in order to observe the standard Unruh result one has to extend the mode profile $g(z)$ near the boundaries, i.e., eliminate nonadiabatic boundary contributions corresponding to the second term in Eq. (6b).

The nonadiabatic nature of the Unruh effect can be demonstrated most clearly by following explicit derivation of the Unruh factor as a probability of the nonadiabatic transition 17] $\psi_{0} \rightarrow \psi_{1}$ from the dressed ground state. Indeed, the Shroedinger equation $i \hbar d \psi / d \tau=$ $H \psi$ in the two-level case $\psi=c_{0} \psi_{0}+c_{1} \psi_{1}$ yields $d c_{1} / d \tau+\left(i E_{1} / \hbar+\left\langle\dot{\psi}_{1} \mid \psi_{1}\right\rangle\right) c_{1}=-c_{0}\left\langle\dot{\psi}_{0} \mid \psi_{1}\right\rangle$. The difference between the eigenenergies is, to the first order, $E_{1}-E_{0}=\hbar\left(\omega+\nu^{\prime}\right)$. For small nonadiabatic coupling $-\left\langle\dot{\psi}_{0} \mid \psi_{1}\right\rangle=\frac{d}{d \tau}\left(\frac{g(\tau)}{\omega+\nu^{\prime}}\right) \ll \omega+\nu^{\prime}$, the perturbation solu- tion is $\left|c_{1}\right|^{2}=\left|\int_{\tau_{i}}^{\tau} \exp \left[i \int_{\tau_{i}}^{\tau^{\prime}}\left(\nu^{\prime}+\omega\right) d \tau^{\prime \prime}\right] \frac{d}{d \tau^{\prime}}\left(\frac{g\left(\tau^{\prime}\right)}{\omega+\nu^{\prime}}\right) d \tau^{\prime}\right|^{2}$. If we now make the assumption of an adiabatic switching (on and off) of the interaction $g(\tau)$ as in standard Unruh effect treatments, then after integration by parts the latter integral is reduced to the integral $I_{2}(\omega)=I_{1}(-\omega)$ in Eqs. (6) but in the infinite limits, i.e. without edge effects. This yields the standard Unruh factor $\left|c_{1}\right|^{2} \propto \exp (-2 \pi \omega / \alpha)$. This derivation clearly shows the dramatic effect of boundary contributions leading to a large amplitude $\sim g(\tau) /\left(\omega+\nu^{\prime}\right)$ of the atomic excited state $|a\rangle$. Only if we eliminate the edge effects by adiabatic switching of the interaction, do we retrieve the exponentially small excitation factor.

The surprising result is that in the cavity, the excitation factor $\exp \left(-\hbar \nu / k_{B} \mathcal{T}_{c}\right) \equiv R_{2} / R_{1}=\alpha / 2 \pi \omega$ is determined by the first power of the same nonadiabaticity parameter $\alpha / 2 \pi \omega$. The reason for this effect is the existence of a true resonance, i.e., a stationary-phase point, in the absorption coefficient (the first term in the integral $I_{1}$ in Eqs. (6b) and (7a)). As mentioned earlier, this yields a resonance between the atomic transition frequency and the Doppler-shifted frequency of the field seen by the atom, $\omega+\frac{d}{d \tau}\left(\frac{\nu}{\alpha} \mathrm{e}^{-\alpha \tau}\right) \simeq 0$, and is responsible for the aforementioned effect.

Another surprise of the cavity acceleration radiation is squeezing. If the atoms are injected at regular intervals of times, $t_{0 i}=\pi m_{i} / \nu+t_{\phi}$, where $m_{i}$ is an integer, all atoms have the same phase with respect to the cavity mode, $\Phi=\sum_{j=1}^{\Delta N} e^{-2 i \pi m_{j}-2 i \nu t_{\phi}} / \Delta N=e^{-2 i \nu t_{\phi}}$, and instead of Eq. (4) we find

$$
\begin{aligned}
& \dot{\rho}_{n, n}=-R_{1}\left[n \rho_{n n}-(n+1) \rho_{n+1, n+1}\right] \\
& -R_{2}\left[(n+1) \rho_{n, n}-n \rho_{n-1, n-1}\right] \\
& +\left[-S_{1} \sqrt{(n+1)(n+2)} \rho_{n+2, n}-S_{2} \sqrt{(n-1) n} \rho_{n, n-2}\right. \\
& \left.+\left(S_{1}+S_{2}\right) \sqrt{(n+1) n} \rho_{n+1, n-1}+\text { h.c. }\right] .
\end{aligned}
$$

In this case the analysis is similar to the analysis of a polarization injected laser [9], and the radiation density matrix is far from being thermal due to squeezing factors

$$
\begin{array}{r}
S_{1,2}=r g^{2} \Phi \mathrm{e}^{-2 i \nu / \alpha} \int_{\tau_{i}}^{\tau_{i}+T} d \tau^{\prime} \int_{\tau_{i}}^{\tau^{\prime}} d \tau^{\prime \prime} \times \\
e^{i \frac{\nu}{\alpha} e^{-\alpha \tau^{\prime}} \mp i \omega \tau^{\prime}-\alpha \tau^{\prime}} e^{i \frac{\nu}{\alpha} e^{-\alpha \tau^{\prime \prime}} \pm i \omega \tau^{\prime \prime}-\alpha \tau^{\prime \prime}} .
\end{array}
$$

It is also possible to implement a more powerful resonant emission by ground state atoms in a cavity, e.g. when the center of mass of the atom is oscillating as $z(\tau)=z_{\mathrm{o}} \cos \left(\omega_{\mathrm{o}} \tau\right)$, Fig. 1(b). This can be viewed as another example of mazer action. In such a case, the density matrix of a cavity mode is again found to obey Eq. (8) but now

$$
R_{1,2} \cong \frac{r g^{2}}{(\gamma+\alpha)^{2}} J_{\mathrm{p}}\left(-k z_{\mathrm{o}}\right) J_{\mathrm{p}}\left(k z_{\mathrm{o}}\right),
$$

where $\mathrm{p}$ is an integer, $J_{p}(x)$ the Bessel function, $\gamma$ the effective atomic decay rate; and the squeezing terms 
$S_{1,2}$ are governed by cross terms which go as $\left(\mathrm{rg}^{2} /(\gamma+\right.$ $\left.\alpha)^{2}\right) J_{\mathrm{p}} J_{0}$. Since this is a resonant parametric process, the absorption $(p=0, \omega=\nu)$ and emission $(p \neq 0$, $\left.\omega+\nu=p \omega_{0}\right)$ coefficients (9) are larger than for counterrotating interactions, Eqs. (6) and (7a), by a resonant factor $[\nu /(\gamma+\alpha)]^{2}$. In this case, parametric generation is possible [18] (see [1] for details).

Concluding remarks. Our simple model clearly demonstrates that the ground state atoms accelerated through a vacuum-state cavity radiate real photons. For relatively small acceleration $a<2 \pi \omega c$, the excitation Boltzman factor $\exp \left(-\hbar \nu / k_{B} \mathcal{T}_{c}\right) \sim \alpha / 2 \pi \omega$ is much larger than the standard Unruh factor $\exp (-2 \pi \omega / \alpha)$. The physical origin of the field energy in the cavity and of the real internal energy in the atom is, of course, the work done by an external force driving the center-of-mass motion of the atom against the radiation reaction force. Both the present effect (in a cavity) and standard Unruh ef- fect (in free space) originate from the transition of the ground state atom to the excited state with simultaneous emission of photon due to the counter-rotating term $\hat{a}_{k}^{+} \hat{\sigma}^{+}$in the time-dependent Hamiltonian (3). The enhanced rate of emission into the cavity mode comes from the second term in Eqs. (6b) and (7a)-the nonadiabatic transition at the boundaries of the cavity; the standard Unruh excitation comes from the first term in Eqs. (6b) and $(7 \mathrm{a})$ - the nonadiabatic transition in free space due to the time dependence of the Doppler-shifted field frequency $\nu^{\prime}=\nu \mathrm{e}^{-\alpha \tau}$, as seen by the atom in the course of acceleration.

The authors gratefully acknowledge the support from DARPA-QuIST, ONR, and the Welch Foundation. We would also like to thank R. Allen, H. Brandt, I. Cirac, J. Dowling, S. Fulling, R. Indik, P. Meystre, W. Schleich, L. Susskind, and W. Unruh for helpful discussions.
[1] S.A. Fulling, Phys. Rev. D7, 2850 (1973); W.G. Unruh, Phys. Rev. D14, 870 (1976); P. Davies, J. Phys. A8, 609 (1975); B.S. DeWitt, in General Relativity: An Einstein Centenary Survey, ed. by S.W. Hawking and W. Israel, Cambridge University Press (1979).

[2] N. Birrell and P. Davies, Quantum Fields in Curved Spacetime, Cambridge Press (1982); W. Unruh and R. Wald, Phys. Rev. D29, 1047 (1984); V.L. Ginzburg and V.P. Frolov, Sov. Phys. Usp. 30, 1073 (1987); A. Barut and J. Dowling, Phys. Rev. A41, 2277 (1990); P. Milonni, The Quantum Vacuum, p. 64 Academic Press (1994); J. Audretsch and R. Müller, Phys. Rev. D49, 4056 (1994); N.B. Narozhny, A.M. Fedotov, B.M. Karnakov, et al., Phys. Rev. D65, 025004 (2001).

[3] For example, the acceleration experienced by $\mathrm{He}^{+}$in a particle acclerator yielding a field $\sim 10^{8} \mathrm{~V} / \mathrm{m}$.

[4] The frequency $\omega$ is lower bounded (for a possible experiment) by cryogenic technology and the requirement that the effect should not be obscured by "hot" walls.

[5] M. Scully, G. Meyer, and H. Walther, Phys. Rev. Lett. 76, 4144 (1996); B.-G. Englert, J. Schwinger, A. Barut, and M. Scully, Europhys. Lett. 14, 25 (1991). We distinguish between the usual stimulated emission maser physics and that characterized by the present " $z$-motion induced" emission and call the process

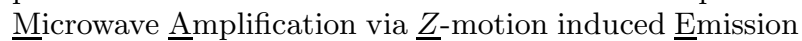
of Radiation "mazer" action.

[6] See also the work of G. Agarwal and coworkers [Phys. Rev. A66, 043812 (2002)] and W. Schleich and coworkers [Phys. Rev. A56, 4164 (1977)] and references therein.

[7] See also the interesting experimental suggestion by E. Yablonovitch, Phys. Rev. Lett. 62, 1742 (1989).

[8] R. Boyd, Nonlinear Optics, Academic Press (1992).

[9] B.-G. Englert, J. Schwinger, and M.O. Scully, in New Frontiers in Quantum Electrodynamics and Quantum Optics, ed. A.O. Barut (Plenum, New York, 1990); see also M. Scully and S. Zubairy Quantum Optics, Cambridge Press (1997) and references therein.

[10] M. Scully et al., to be published.

[11] V. Kocharovsky et al., to be published.
[12] For the density matrix quantum theory of the laser see M. Scully and W. Lamb Jr., Phys. Rev. Lett. 16, 853 (1966). For pedagogical treatment and references see Pike and Sakar The Quantum Theory of Radiation, Oxford University Press (1997) or M. Scully and S. Zubairy Quantum Optics, Cambridge Press (1997).

[13] For the quantum analysis of the micromaser relevant to the present problem see P. Filipowicz, J. Javanainen and P. Meystre, J. Opt. Soc. Am. B3, 906 (1986).

[14] W. Rindler Essential Relativity, Springer-Verlag (1977) and references therein for a discussion of "Rindler coordinates".

[15] For brevity, we keep only the main terms in the expressions for the eigenstates. For details, see, e.g., S. Swain, J. Phys. A6, 1919 (1973).

[16] The Doppler-shifted frequency of the field, as it is seen by the atom is $\nu^{\prime}=d\left(\nu t(\tau)-k_{z} z(\tau)\right) / d \tau=\nu \mathrm{e}^{-\alpha \tau}$. For the case of a counter-propagating wave one has to change the sign of $\alpha$ in the $\mathrm{e}^{-\alpha \tau}$ and related Eq. (6).

[17] For the review on nonadiabatic transitions see, e.g., V.V. Zheleznyakov, V.V. Kocharovsky, and Vl.V. Kocharovsky, Sov. Phys. Usp. 26, 877 (1983).

[18] Remarkably, as the analysis of the result (6) shows, parametric gain is possible in cavity QED via counterresonant emission by ground state atoms with random injection times. For such gain to occur, the time of flight $T$ should be tuned to a set of specific values to ensure that the atom emits into the cavity mode more energy than it takes away, $R_{2}>R_{1}$. The counter-propagating mode is more favorable for gain since the absorption can then be anomalously small while the gain remains as large as for the co-propagating mode. The gain can be easily seen in the case of a constant velocity:

$$
\frac{R_{2}}{R_{1}}=\left|\frac{\nu^{\prime}-\omega}{\nu^{\prime}+\omega}\right|^{2}\left|\frac{1-e^{-i\left(\nu^{\prime}+\omega\right) T}}{1-e^{-i\left(\nu^{\prime}-\omega\right) T}}\right|^{2}, \nu^{\prime}=\nu\left(\frac{\nu-\mathbf{k} \cdot \mathbf{v}}{\nu+\mathbf{k} \cdot \mathbf{v}}\right)^{1 / 2}
$$

Namely, one should tune the time of flight to get the proper interference factors: $e^{-i\left(\nu^{\prime}-\omega\right) T} \rightarrow$ 
1, $\left|e^{-i\left(\nu^{\prime}+\omega\right) T}-1\right| \sim 1$. A similar time of flight tuning is used in some electronic devices, e.g., klystrons. 